\title{
Mice lacking global Stap1 expression do not manifest hypercholesterolemia
}

\author{
Babunageswararao Kanuri ${ }^{1}$, Vincent Fong ${ }^{1}$, April Haller ${ }^{2}$, David Y. Hui ${ }^{2}$ and Shailendra B. Patel ${ }^{1 *}$ (D)
}

\begin{abstract}
Background: Autosomal dominant familial hypercholesterolemia (ADH; MIM\#143890) is one of the most common monogenic disorders characterized by elevated circulatory LDL cholesterol. Initial studies in humans with ADH identified a potential relationship with variants of the gene encoding signal transducing adaptor family member protein 1 (STAP1; MIM\#604298). However, subsequent studies have been contradictory. In this study, mice lacking global Stap1 expression (Stap ${ }^{-/-}$) were characterized under standard chow and a $42 \% \mathrm{kcal}$ western diet (WD).

Methods: Mice were studied for changes in different metabolic parameters before and after a 16-week WD regime. Growth curves, body fats, circulatory lipids, parameters of glucose homeostasis, and liver architecture were studied for comparisons.

Results: Surprisingly, Stap ${ }^{-/-}$mice fed the 16-week WD demonstrated no marked differences in any of the metabolic parameters compared to Stap $1^{+/+}$mice. Furthermore, hepatic architecture and cholesterol content in FPLC-isolated lipoprotein fractions also remained comparable to wild-type mice.

Conclusion: These results strongly suggest that STAP1 does not alter lipid levels, that a western diet did not exacerbate a lipid disorder in Stap1 deficient mice and support the contention that it is not causative for hyperlipidemia in ADH patients. These results support other published studies also questioning the role of this locus in human hypercholesterolemia.
\end{abstract}

Keywords: STAP1, Autosomal dominant familial hypercholesterolemia, Familial hypercholesterolemia 4, B-cells, Western diet, Fast performance liquid chromatography

\section{Background}

Familial hypercholesterolemia (FH) is a widely prevalent congenital metabolic disorder characterized by substantially elevated circulatory low density lipoprotein cholesterol (LDL-C) and accelerated cardiovascular events $[1,2]$. Autosomal dominant inherited hypercholesterolemia $(\mathrm{ADH})$ accounts for a major proportion of the FH cases worldwide with mutations most commonly reported in low density lipoprotein receptor $(L D L R)$, apolipoprotein $\mathrm{B}$ $(A P O B)$, and proprotein convertase subtilisin/kexin type 9 (PCSK9) [3-5]. However, a number of patients classified

\footnotetext{
*Correspondence: sbpatel@uc.edu

'Division of Endocrinology, Diabetes and Metabolism, University of Cincinnati, Cincinnati, OH, USA

Full list of author information is available at the end of the article
}

as having familial hypercholesterolemia 4 (FH4), demonstrate the ADH phenotype despite having no mutations in these genes [6]. Initial studies involving these patients reported mutations in genes coding for signal-transducing adaptor family member 1 (STAP1) [6-8].

STAP1 (MIM\#604298), also called B-cell antigen receptor downstream signaling 1 protein (BRDG1) or stem cell adaptor protein 1 , was first discovered in immune cells with the highest expression documented in appendix, lymph nodes, and spleen [7]. The gene encoding STAP1 protein is located on chromosomal region 4q13.2 with 10 exons in the protein coding region [7]. Its relationship to lipid homeostasis was first suggested in 2007 in a clinical study finding a correlation between increased levels of circulatory triglycerides and STAP1 gene expression in

C C The Author(s). 2020 Open Access This article is licensed under a Creative Commons Attribution 4.0 International License, which permits use, sharing, adaptation, distribution and reproduction in any medium or format, as long as you give appropriate credit to the original author(s) and the source, provide a link to the Creative Commons licence, and indicate if changes were made. The images or other third party material in this article are included in the article's Creative Commons licence, unless indicated otherwise in a credit line to the material. If material is not included in the article's Creative Commons licence and your intended use is not permitted by statutory regulation or exceeds the permitted use, you will need to obtain permission directly from the copyright holder. To view a copy of this licence, visit http://creativecommons.org/licenses/by/4.0/ The Creative Commons Public Domain Dedication waiver (http://creativecommons.org/publicdomain/zero/1.0/) applies to the data made available in this article, unless otherwise stated in a credit line to the data. 
leukocytes [9]. Later, in 2014, Fouchier and colleagues discovered that variants of the STAP1 gene were associated with the FH phenotype in a linkage analysis of a Dutch family with $\mathrm{FH} 4$ and suggested robust support for genetic causality [6]. Subsequent studies reported STAP1 gene variants observed in such $\mathrm{FH}$ patients also manifested significant cardiovascular events [10-12]. However, other studies found no association between STAP1 variants and hypercholesterolemia [13-16]. An analysis of seven families with $\mathrm{FH}$ phenotype failed to observe the co-segregation of four rare predicted pathogenic variants of STAP1 [17]. Loaiza et al more recently demonstrated no marked changes plasma lipid profiles of carriers of STAP1 variants compare to controls as well as in a mouse model [18]. Therefore, we sought out to understand the STAP1 role by using a mouse knockout model, to ask if loss of STAP1 would lead to hyperlipidemia.

In this study, mice lacking global expression of Stap1 gene $\left(S t a p 1^{-/-}\right)$were characterized for various metabolic parameters on standard diet and after 16 weeks of western diet (WD) regime. The present work presents an independent characterization of Stap1 ${ }^{-/-}$mice to that studied by Loaiza et al with some differences between models and confirms the argument against STAP1 having a role in causing $\mathrm{FH}$.

\section{Methods}

\section{Knockout mice and diets}

Heterozygous Stap1 KO mice $\left(\right.$ Stap $\left.^{+/-}\right)$on C57BL/6 N background generated through reporter-tagged insertion were imported from Wellcome Trust Sanger Center, via EUCOMM [19-22]. The 'knockout first' tm1a (Stap1 ${ }^{\text {t- }}$ mla(KOMP)Wtsi $)$ mutation bears the IRES:lacZ trapping cassette and a floxed promoter-driven neo cassette inserted into the intron of the gene in order to disrupt the gene function [22]. Brother-sister matings of the heterozygotes generated homozygous knockout mice $\left(\right.$ Stap $\left.1^{-/-}\right)$and corresponding WT $\left(\right.$Stap $\left.^{+/+}\right)$littermate controls. Tail snip genotyping was performed to assess the genotype of each mouse before recruiting into experiments; amplimers of $266 \mathrm{bp}$ and $236 \mathrm{bp}$ correspond to $\mathrm{Stap}^{+/+}$and Stap $1^{-/-}$, respectively (Table). Mice were fed with irradiated standard rodent-chow diet (CD; Envigo 7912) [23] and water ad libitum, with equal light and dark cycles. Baseline measurements were performed in 10 to 11 week-old male and female mice on rodent chow and then placed on a high-cholesterol western diet (WD; Envigo TD.88137) for another 16 weeks [24]. Chow and western diets provided 17 and $42 \% \mathrm{kcal}$ energy from fats $[23,24]$ and were procured from Harlan Teklad, Madison, WI, USA. All animal studies were approved by the Institutional Animal Care and Use Committee at the University of Cincinnati (Protocol No.
16-04-19-01). Terminal blood samples were obtained under full inhaled isoflurane anesthesia. For all studies, except where indicated, each group consisted of 6 mice, based upon prior data that show the power to detect $20 \%$ differences for the most variable procedure (OGTT) where the Power needed is $n=5$, but we included and extra mouse to ensure Power was not due to loss of a mouse for any inadvertent loss. The investigators were not blinded to the samples or mouse genotypes, as there are no datasets collected which required subjective analyses. However, all mice were group housed and each cage contained mice of both genotypes to minimize bias and variation. Mice were sacrified by $\mathrm{CO} 2$ inhalation, followed by cervical dislocation and bilateral thoracotomy.

\section{Measurement of whole-body composition}

Whole-body fat and lean mass were measured in live mice using ECHOMRI ${ }^{\mathrm{Im}}-100 \mathrm{H}$ whole body composition analyzer (Echo Medical Systems, Texas, USA) according to the manufacturer's specifications. Absolute weights (g) of fat and lean mass of each mouse were used to calculate their \% changes [(absolute fat or lean mass weight $(\mathrm{g}) /$ whole body weight $\left.(\mathrm{g}))^{*} 100\right]$, a widely employed method to determine the diet induced obesity phenotype.

\section{Oral glucose tolerance test (OGTT)}

For the OGTT experiment, the experimental mice were initially acclimatized in the procedure room overnight followed by $4 \mathrm{~h}$ fast period in the next day morning. After baseline blood glucose measurements, 50\% Dextrose (Cat\# D16-1; ThermoFisher) in $0.9 \%$ saline was administered via oral gavage at a dose of $2 \mathrm{~g} / \mathrm{kg}$ of body weight [24]. Time dependent changes in blood glucose were measured via tail vein bleeds at $0,15,30$ and 60 min using Accu-Chek Nano electronic glucometer (Roche Applied Science, Indianapolis, IN, USA).

\section{Fast performance liquid chromatography (FPLC)}

Lipoprotein separation for total cholesterol estimation in plasma samples was performed using FLPC as described previously [24]. Briefly, blood was collected from $4 \mathrm{~h}$ fasted mice through the submandibular plexus and then centrifuged at $2500 \times \mathrm{g}$ for $10 \mathrm{~min}$ at $4{ }^{\circ} \mathrm{C}$. The isolated plasma was then measured for basal total cholesterol to exclude any outliers (to ensure pooling was performed between comparable samples) and $200 \mu \mathrm{L}$ of pooled plasma from each group was loaded onto the Akta pure FPLC to separate different lipoproteins by column chromatography (10/300GL, superpose 6). A total of 52 fractions with volume of $500 \mu \mathrm{L}$ each were collected and analyzed for cholesterol content using an enzymatic kit as detailed below. 


\section{Plasma biochemistry}

Plasma triglycerides and cholesterol were estimated using Infinity Triglyceride (TR22421) and Cholesterol (TR13421) calorimetric kits (Thermo Fisher Scientific, Middletown VA, USA) per manufacturer instructions. For estimation of tissue lipids after the western diet phase, livers from Stap $1^{+/+}$and Stap $1^{-/-}$mice were homogenized, normalized for equal protein content, and then measured for total cholesterol and triglycerides using the same calorimetric kits used for plasma samples.

\section{Tissue harvesting and histology of liver}

Mice were euthanized for terminal tissue collection at the end of 16 weeks WD regime. Four-hour pre-fasted mice were euthanized by $\mathrm{CO}_{2}$ exposure and bilateral thoracotomy. Liver, spleen, and kidneys were blotted dry, weighed, and then flash frozen in liquid $\mathrm{N}_{2}$. A small portion of liver was rinsed in chilled PBS (pH -7.4) and fixed in 10\% neutral buffer formalin (5725; Fisher Scientific, Pittsburg, PA, USA) for paraffin embedding and hematoxylin and eosin (H\&E) staining [25]. About $5 \mu \mathrm{m}$ of paraffin embedded sections were cut, processed in graded volumes of ethanol, and then stained with $H \& E$. Images of the stained liver sections were captured at $20 \mathrm{x}$ and 40x objective lens magnification using Olympus BX61 microscope.

\section{QPCR analysis of gene expression}

To determine the tissue expression profile of Stap1, total RNA were isolated from different tissues of Stap $1^{+/+}$mice using Qiagen RNeasy Mini Kit (74,104; Qiagen Inc., Germantown, MD, USA). Isolated RNAs were reverse transcribed to cDNA using SuperScript First-Strand Synthesis kit (11904-018; Invitrogen, Carlsbad, CA, USA) and then assessed for the Stap1 gene expression on Applied Biosystems 7300 Real-Time PCR system (Applied Biosystems, Foster City, CA, USA). Each sample was run in triplicates using power SYBR Green PCR master mix (4,367,659; Applied Biosystems, Foster City, CA, USA). Comparative $C_{T}$ method was used for quantitation post data normalization to Atp5po expression as the house keeping gene to compensate the variations between input RNA amounts [25]. The list of different primers used for PCR experiments was tabulated in the Table S1 (Additional file 1).

\section{Statistical analyses}

An unpaired t-test (without or without Welch's corrections) or Mann-Whitney U test was performed to assess significant between Stap $^{-/-}$and Stap1 ${ }^{+/+}$mice within each sex.

\section{Results}

\section{Validation of Stap1 gene deletion}

The targeting strategy for generation of global Stap1 knockout mice is depicted in Fig. 1a. Measurement of transcript levels of Stap1 in WT mice by qPCR revealed gene expression in a variety of tissues with the highest levels demonstrated in spleen (Fig. 1b). The complete absence of Stap1 expression was confirmed with qPCR in Stap $^{-/-}$mice liver, kidney, and spleen (Fig. 1c). RTPCR analysis of exons 2-5, 2-6, and 2-7 using total RNA isolated from livers and spleens demonstrated sequential exon splicing of exons 5, 6, and 7 in Stap $1^{+/+}$ mice and no products in Stap1 ${ }^{-1-}$ mice (Fig. 1d). The single slightly larger amplicon for exon 2-7 in Stap1 $1^{-/}$ spleen (Fig. 1d, open arrowhead) was sequenced and demonstrated an off-target product on chromosome 2 (Additional file 2).

\section{Baseline characteristics}

Baseline measurements on standard chow diet were assessed at 10-11 weeks of age and body weights (Fig. 3a), whole-body fat (Fig. 2a) and lean mass (Fig. 2a) were similar between Stap $^{+/+}$and Stap $^{-/-}$mice. Further biochemical assessment found no significant differences in glucose tolerance (Fig. 2b), plasma total cholesterol (Fig. 2c), or plasma triglycerides (Fig. 2d) between knockout mice and wildtype mice. Lipoprotein cholesterol profiles in male knockout and wild-type mice were analyzed by FPLC (Fig. 2e) and were also found to be unaffected by the loss of Stap1.

\section{Effects of western diet (WD)}

To determine the role of Stap1 under metabolic challenge, mice were fed western diet $(42 \% \mathrm{kcal}$ from fat). Prior to the commencement of the western diets, baseline body weights showed no statistical differences between wildtype and knockout mice $\left(\right.$ Stap $^{+/+}$males $25.2 \mathrm{~g}+/-3.2$ vs Stap $1^{-/-}$males $23.5+/-3.1$. Stap $1^{+/+}$ females $22.5+/-2.6$ vs Stap $1^{-/-}$females $19.7+/-1.3$, $n=6$ for each group). Body weights were measured weekly (Fig. 3a), while body composition (Fig. 3b) and tissue weights (Fig. 3c and d) were assessed the end of the WD. No significant differences were noted between Stap $1^{-/-}$mice over Stap $1^{+/+}$littermate controls in any of these parameters. Similar trends were also observed in glucose tolerance testing, monitored to ensure there were no differential changes in intermediary metabolism induced by the western diet and loss of Stap1 (Fig. 3e).

Plasma cholesterol and triglyceride levels (Fig. 4a and b), and plasma FPLC cholesterol profiles (Fig. 4c and d) showed the diets led to a significant change in the lipoprotein distribution from baseline (compare to Fig. 2e) as expected for a high fat diet, but there were no differences between wild-type and Stap1 knockout mice.

Investigation of liver histology with hematoxylin and eosin staining (H\&E) showed changes compatible with fat accumulation as would be expected after 16 weeks of WD, but found no differences in morphology between 


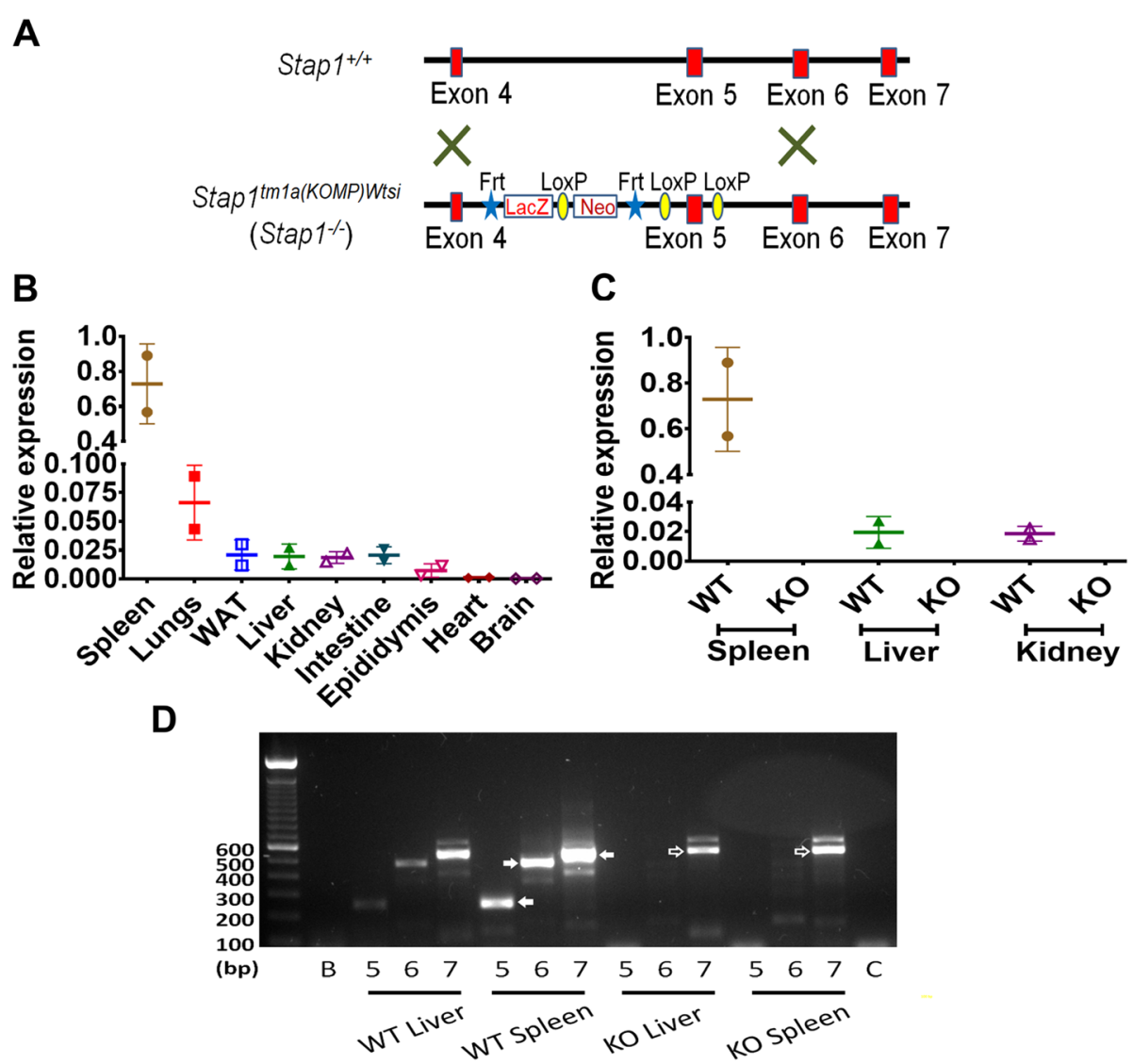

Fig. 1 Verification of global deletion of Stap1 gene expression. Panel a shows the gene targeting details for the generation of Stap $1^{-/-}$mice. This construct is a 'knockout-first', which can be converted to a conditional, upon deletion of the LacZ-Neo cassette. The mice used in this study remain as knockout and retain this cassette. A tissue survey of Stap1 mRNA was performed using qPCR in tissues collected from Stap $1^{+/+}$mice (panel $\mathbf{b}, n=2$ ). Note that compared to a house-keeping gene expression (see Methods), only spleen and lung showed some expression with very low levels detected in other tissues. Stap $1^{-1-}$ mice (KO) showed no detectable qRT-PCR products (panel $\mathbf{c}, \mathrm{n}=2-3$ ) compared to wild-type mice (WT) in spleen, liver or kidney. To further confirm a lack of legitimate transcript expression, RT-PCR analysis of exon splicing for exons $2-5$, 2-6, and 2-7 in liver and spleen was performed (panel $\mathbf{d}$ ). The labels ' 5,6 , and 7 ' for each tissue indicates the location of the reverse primers targeting exons 5, 6, or 7 respectively, used with a forward primer located in exon 2. Spleen mRNA from WT tissues amplified the correct expected size of product and size increments (exon 2-5; 251 bp, exon 2-6; 482 bp, and exon 2-7; 536 bp indicated by filled arrowheads). No products were noted when exons 2-5, or 2-6 were used for KO spleen mRNA. A slightly larger product was noted for exons 2-7 (indicated by open arrowhead), though on sequencing this was found to be a non-specific product from mis-priming (see Text). Error bars denote \pm 1 SD

groups (Fig. 5a and b). Moreover, the elevated levels of liver total cholesterol and triglycerides (Fig. $5 \mathrm{c}$ and d) were comparable between WD fed Stap1 ${ }^{+/+}$and Stap1 ${ }^{-/-}$mice.

\section{Discussion}

This study tested the hypothesis that loss of Stap1 can lead to hypercholesterolemia using Stap $1^{-/-}$mice. The Stap 1 mice, targeted by a knockout-first, conditional targeting vector were obtained from the Welcome Trust Sanger Center and characterized. Although there is no reliable antibody for specific detection of endogenous STAP1 protein by western blot (we tested commercial antibodies from ThermoFisher and Abcam but found these unsuitable, see Supplementary files), a knockout was confirmed at the DNA and mRNA level instead. Normal Stap1 RNA expression observed in different tissues of $\operatorname{Stap}^{+/+}$mice and compared with the protein atlas database (https://www.proteinatlas.org/ENSG0000 0035720-STAP1/tissue); relatively high levels of expression in spleen were noted but very minimal expression was seen in liver. The higher levels of RNA also coincide with the proportional amount of STAP1 protein in spleen of Stap1 ${ }^{+/+}$mice as measured through targeted proteomics [18]. QPCR analysis of livers, spleens, and kidneys of Stap $^{-1-}$ mice did not detect any Stap1 mRNA, confirming the knockout (Fig. 1c). RT-PCR analysis of the mRNA produced expected amplicons when using primers targeted to exon 2 through 4 in Stap1 ${ }^{-/-}$ mice (data not shown), but not with primers specific for exons 5, 6 or 7 (Fig. 1d) suggestive that the transcriptional message was preserved until exon 4 and was interrupted due to reporter insertion in the intronic region 
A

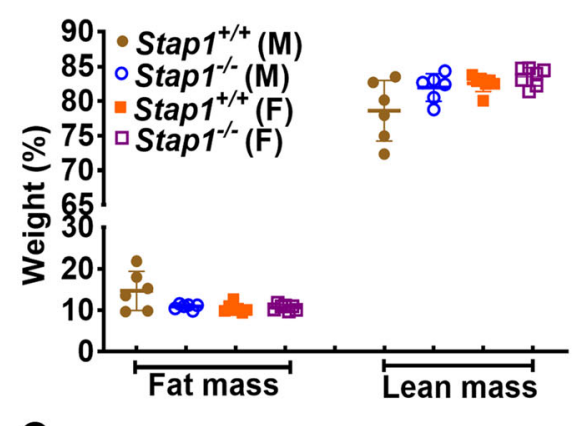

C

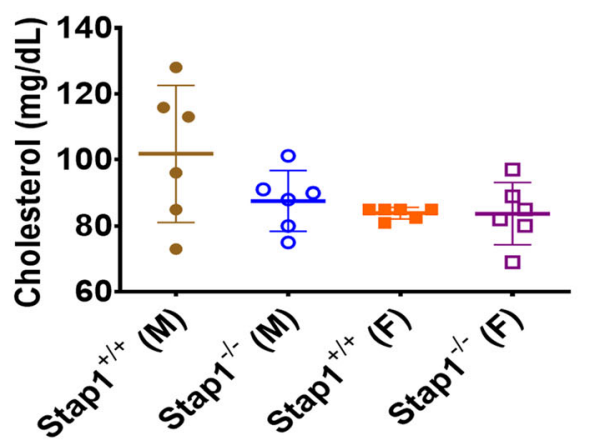

E
B

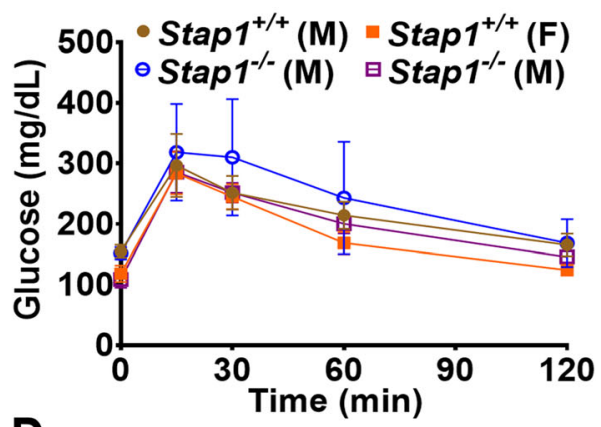

D

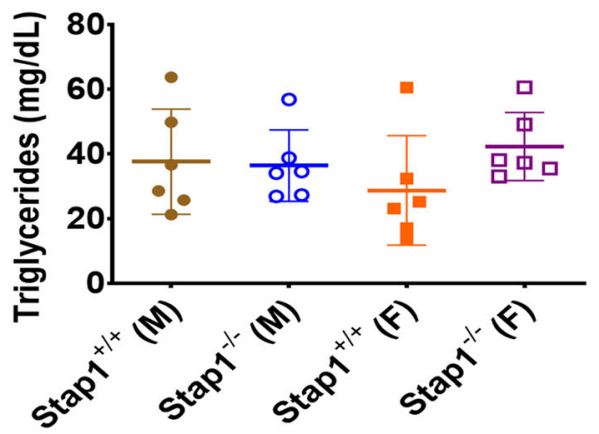

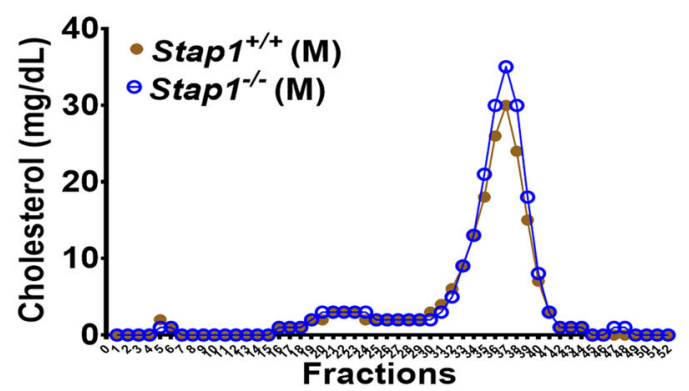

Fig. 2 Baseline metabolic parameters in chow-fed Stap $1^{-1-}$ and control mice. Panel a shows the starting body composition of mice at age 5-6 weeks on a chow diet $(n=6-7)$. Starting lean or fat masses between wildtype and knockout mice were comparable. Glucose tolerance (Panel $\mathbf{b}$, $n=6$ ), plasma cholesterol (Panel $\mathbf{c}, n=6$ ), triglycerides (Panel $\mathbf{d}, n=6$ ) were also comparable and showed no statistical differences. Panel e shows the FPLC cholesterol profiles in 10-11 weeks old male Stap $1^{+/+}$and Stap $1^{-1-}$ mice and was also indistinguishable. Note that the knockout mice do not show any differences in the VLDL (fractions 3-6), or LDL (fractions 20-28) ranges. Error bars denote \pm 1 SD

between exon 4 and 5. RT-PCR using primers targeted to exon 2 through 7 unexpectedly produced a product in Stap $1^{-/-}$mice (Fig. 1d), but sequencing showed that it was an off target product from chromosome 2 (supplemental data), whereas Stap1 is located on chromosome 4.

Obesity and insulin resistance are commonly associated with hyperlipidemia and high caloric fat- enriched diets are widely accepted to induce hyperlipidemia [26]. A western-type diet (TD.88137) was chosen for these studies for its ability to induce hypercholesterolemia, obesity and atherosclerosis depending on the genetic background of the mice used for study [24, 27, 28]. Previous studies have shown that 16 weeks of WD regime is sufficient to induce severe hypercholesterolemia in mice $[29,30]$. Augmentation of whole-body fat mass and markedly elevated circulatory cholesterol (especially males) was observed in Stap1 ${ }^{+/+}$and Stap1 ${ }^{-/-}$mice at the end of 16 weeks WD (Figs. 3 and 4). However, these changes were not exacerbated, as hypothesized, in mice lacking global Stap1 expression and suggest the loss of Stap1 does not exacerbate hyperlipidemia.

The link between STAP1 and hypercholesterolemia was proposed when some FH4 patients were reported to have variants of the gene, in the absence of changes in known hyperlipidemia loci [6]. A review of genetic variants associated with FH noted 2580 in $L D L R, 896$ in 


\section{A}

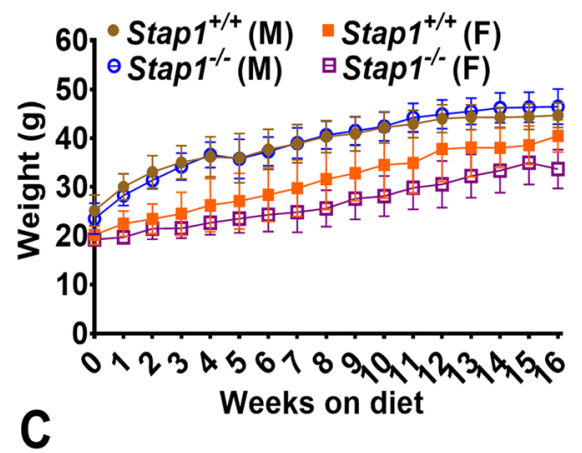

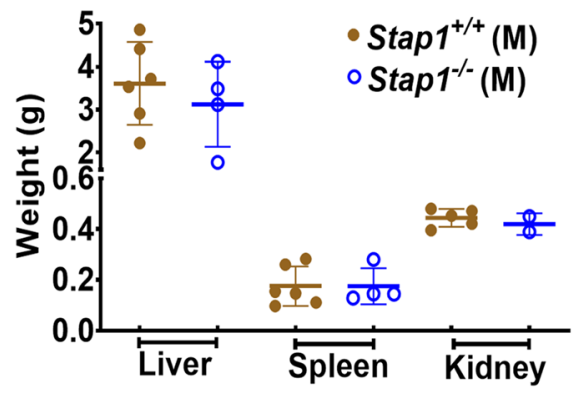

B

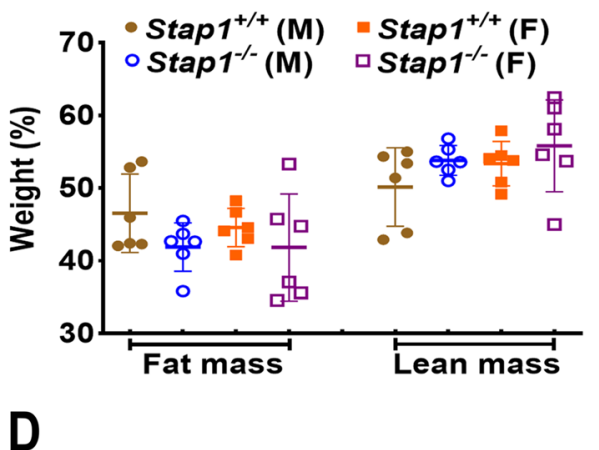

D

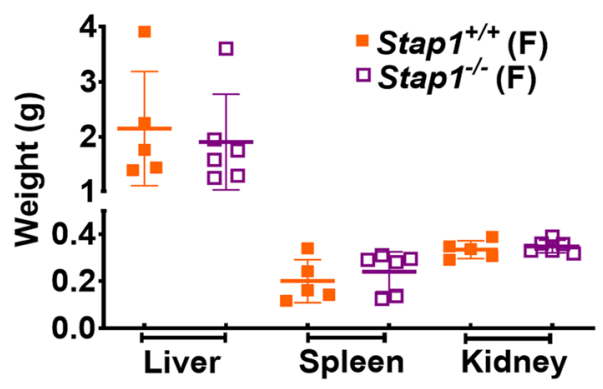

E

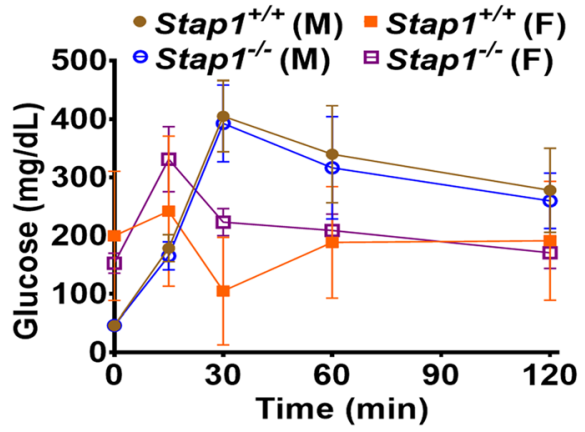

Fig. 3 Effect of western diet on growth parameters and body composition in mice lacking Stap 1 gene. Panel a shows the growth curves in wildtype and knockout mice and no statistical differences were discernable in the patterns of weight gain on the high caloric diet $(n=6)$. Neither the percent fat or lean mass were altered in Stap $1^{-1-}$ mice compared to age and sex-matched wildtype mice on a western diet $($ panel $\mathbf{b}, n=6)$. No changes in weights of liver, spleen or kidneys were seen in male (panel $\mathbf{c}, n=4-6$ ) or female mice (panel $\mathbf{d}, n=5-6$ ) were noted. No changes in glucose tolerance tests were noted between knockout and wildtype controls either (panel e, $n=6$ ). In case of male Stap $1^{-/-}$mice, kidney weights of only 2 mice were considered for analysis

$A P O B, 351$ in PCSK9, 16 in APOE, and 4 in STAP1 [8]. Fouchier et al in 2014 demonstrated four variants in the STAP1 gene of FH4 patients and characterized the phenotype. All four STAP1 variants were missense mutations (Glu97Asp, Leu69Ser, Ile71Thr, and Asp207Asn). Together with the fact that the phenotype of STAP1 variant carriers was milder than other forms of ADH [6], one possibility is whether these are 'false positive' hits. Nevertheless the association between STAP1 and ADH was supported further by other studies showing elevated circulatory cholesterol in patients with STAP1 variants [10-12, 31]. However, this link has been highly debated with studies reporting no association between STAP1 and $\mathrm{ADH}$ [13-17], or no difference in plasma lipid profiles in carriers of STAP1 variants and controls [18]. Moreover, Loaiza et al recently also found the absence of any significant difference in lipid phenotype in Stap $1^{-/-}$mice compared to controls, even after metabolic challenges [18]. The current study is a verification of these findings. Nevertheless, a $\mathrm{KO}$ mouse model is a loss of function model and does not address the possibility of a gain of function variants in Stap1. The fact that the previously identified STAP1 variants were missense mutations suggests that a viable protein may potentially be expressed in those patients, though its functionality would be purely speculative. 

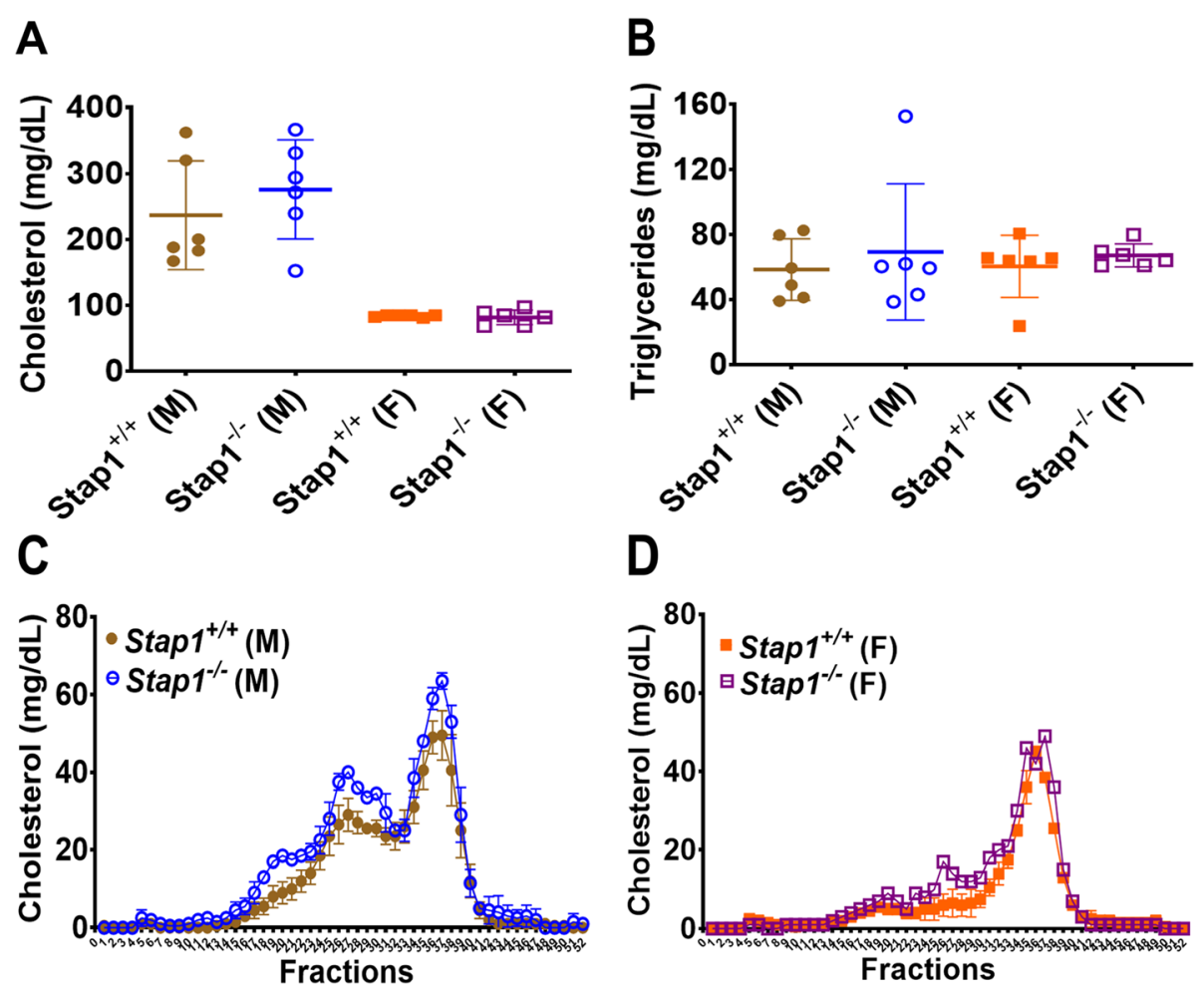

Fig. 4 Effect of 16 weeks of western diet (WD) on plasma lipid profiles. Panel a shows the plasma cholesterol and panel $\mathbf{b}$ the triglyceride levels in wildtype and knockout mice ( $n=6$ per group). Female mice (whether wild-type or knockout) seem to be resistant to a WD-induced hypercholesterolemia (panel a), but this may be a feature of the mouse strain background. FPLC cholesterol profiles of the plasma (panel $\mathbf{c}$, males, panel $\mathbf{d}$, females) showed that the diet in males led to a dyslipidemic profile, but loss of Stap1 did not affect these patterns. Error bars denote \pm 1 SD

It was initially hypothesized that extra-hepatic mechanisms were responsible for STAP1 mediated hypercholesterolemia due to the fact that liver distribution was low [6, 31]. Interestingly, recent experiments using single cell RNA-Seq indicated that its expression is highly specific to lymphoid cells (https://tabula-muris.ds.czbiohub. org/) and any expression detected by examining tissue RNA likely comes from the presence of these cells in these tissues. Stap 1 expression measured by qPCR in the current study was low in liver and other tissues except for the spleen (Fig. 1b). This could be explained by the concentration of B-cells in the spleen and their presence in other organs, as the tissues were not perfused prior to collection. Some studies have associated STAP1 variations with cardiovascular disease [10-12], and immune cells have been linked with atherosclerosis and FH [32]. This study did not thoroughly investigate possible effects of STAP1 deletion on the immune system, but there were no obvious changes in B-cell subsets and IgG (data not shown). Further studies would be obviously necessary to fully characterize the effects, if any, on immune function. Nevertheless, transplant of Stap1 ${ }^{-/-}$bone marrow (BM) into $\mathrm{Ldlr}^{-1-}$ female mice had no impact on plasma lipids as well on the development of atherosclerosis plaques [18]. Furthermore, there were no marked changes in B-lymphocyte populations in blood of carriers of STAP1 gene variants compared to age- and sex-matched family controls [18].

The study by Loaiza et al was well-designed and thorough, but differs from the current investigation in two important experimental variables: 1 ) the strategy used to generate Stap1 KO mice, using clustered regularly interspaced short palindromic repeats/clustered regularly interspaced short palindromic repeat-associated 9 (CRIS PR/Cas9) technology vs targeted homologous recombination of embryonic stem cells and 2) the much shorter time period for hyperlipidemia induction in global and tissue-specific KO mice (4 weeks vs 12 weeks). Since 16 weeks of WD regime is sufficient to induce severe hypercholesterolemia and the fact that FH patients show elevated levels of plasma cholesterol, the long-term regime that we used is ideally advantageous over the short duration regime used in the study by Loaiza et al.

An important limitation of the study is loss-of-function models do not recapitulate the missense variants reported for STAP1 in humans. However, we are confident our current studies are valid as our characterization (in keeping with published data) finds no meaningful expression of STAP1 in cells other than B-cells. Thus, even for missense variants of STAP1 to have a pathological role in 
A

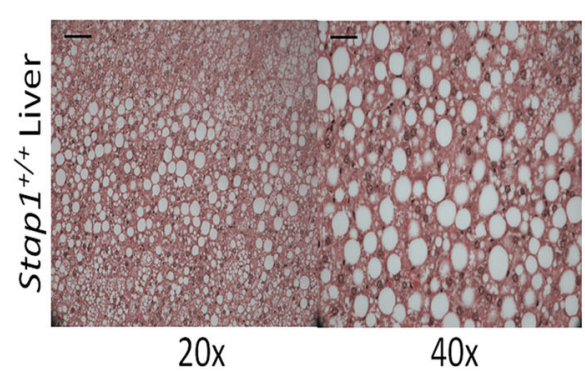

C

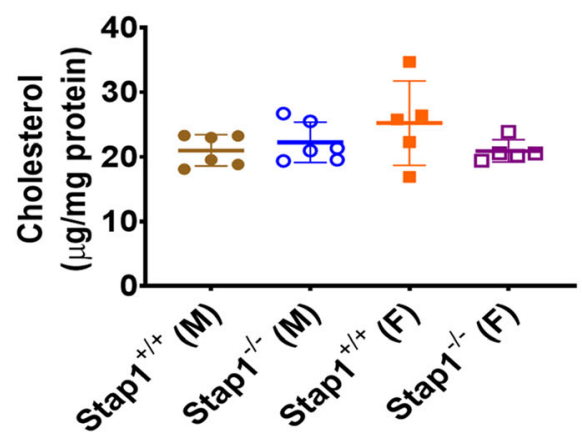

B

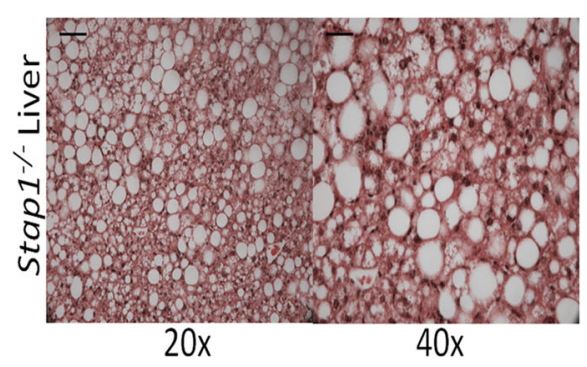

D

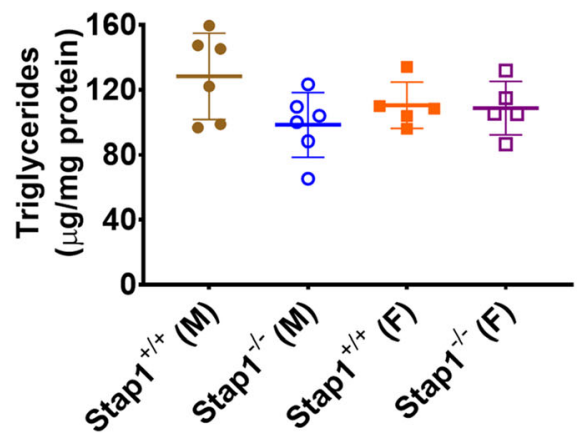

Fig. 5 Effect of western diet (WD) on liver morphology and lipids. Assessment of liver histology by hematoxylin and eosin staining showed a fatty liver in all mice, irrespective of Stap1 status or sex (panel $\mathbf{a}$ and $\mathbf{b}$ ). This was confirmed by analyses of liver cholesterol (panel $\mathbf{c}, \mathrm{n}=6$ per group in males and 5 per group in females) and triglyceride (panel $\mathbf{d}, n=6$ per group in males and 5 per group in females) but loss of Stap 1 does not affect these parameters. Error bars denote \pm 1 SD

causing hyperlipidemia in humans, a mechanistic pathway to do so would need to be identified. Based upon our current understanding of lipid metabolism, no such meaningful pathway exists.

\section{Conclusion}

Global loss of Stap1 in mice does not result in an abnormal lipid phenotype, and this study validates the study by Loaiza et al, who reported similar findings. Additionally, expression STAP1 mRNA seems to be confined to B-cells, suggesting alteration of lipid metabolism in a direct manner to be not feasible. Genetic changes in STAP1 are therefore unlikely to be cause of familial hypercholesterolemia and variants reported in such rare families may represent a spurious association.

\section{Supplementary Information}

The online version contains supplementary material available at https://doi. org/10.1186/s12881-020-01176-X.

Additional file 1: Table S1. Primer sequences.

Additional file 2. Sequencing of off-target products on chromosome 2. Additional file 3.

\section{Abbreviations}

ADH: Autosomal dominant familial hypercholesterolemia;

APOE: Apolipoprotein E; BRDG: B-cell antigen receptor downstream signaling 1 protein; FLPC: Fast Performance Liquid Chromatography; LDLR: Low density lipoprotein receptor; OGTT: Oral glucose tolerance test; PCR: Polymerase chain reaction; PCSK9: Proprotein convertase subtilisin/kexin type 9; STAP1: Signal transducing adaptor family member protein 1; WD: Western diet

\section{Acknowledgments}

We would like to acknowledge the technical assistance of Vicky Horton, Casey Crane, Min Jiang, Ryan Ulrich, and William Thompson.

\section{Authors' contributions}

SBP and DYH conceptualized, designed and supervised the study; $\mathrm{AH}$ performed the experiments; BK and VF analyzed the data and drafted the manuscript. All authors read and approved the final manuscript.

\section{Funding}

This study was supported by institutional funds from the University of Cincinnati (SBP).

\section{Availability of data and materials}

The datasets used and analyzed during the current study are available from the corresponding author upon request. Requests for mice will require a simple materials transfer agreement with the University of Cincinnati and all costs for transfer will be the responsibility of the requestor. 


\section{Ethics approval and consent to participate}

There are no clinical studies involved in this work. All animal studies were approved by the Institutional Animal Care and Use Committee at the University of Cincinnati (Protocol No. 16-04-19-01).

\section{Consent for publication}

All the authors have read and consented for publication of this article.

\section{Competing interests}

The authors declare no competing interests.

\section{Author details}

'Division of Endocrinology, Diabetes and Metabolism, University of Cincinnati, Cincinnati, OH, USA. ${ }^{2}$ Department of Pathology, University of Cincinnati, Cincinnati, OH, USA.

Received: 27 August 2020 Accepted: 15 November 2020 Published online: 23 November 2020

\section{References}

1. Ezhov M, Bazhan S, Ershova A. Clinical guidelines for familial hypercholesterolemia. J Atherosclerosis Dyslipidemias. 2019;1:5-43.

2. Goldberg AC, Hopkins PN, Toth PP, Ballantyne CM, Rader DJ, Robinson JG, Daniels SR, Gidding SS, de Ferranti SD, Ito MK, et al. Familial hypercholesterolemia: screening, diagnosis and management of pediatric and adult patients: clinical guidance from the National Lipid Association Expert Panel on familial hypercholesterolemia. J Clin Lipidol. 2011;5(3 Suppl):S1-8.

3. Kanehisa M, Sato Y, Furumichi M, Morishima K, Tanabe M. New approach for understanding genome variations in KEGG. Nucleic Acids Res. 2019;47(D1): D590-5.

4. lacocca MA, Hegele RA. Recent advances in genetic testing for familial hypercholesterolemia. Expert Rev Mol Diagn. 2017;17(7):641-51.

5. Gidding SS, Champagne MA, de Ferranti SD, Defesche J, Ito MK, Knowle JW, McCrindle B, Raal F, Rader D, Santos RD, et al. The agenda for familial hypercholesterolemia: a scientific statement from the American Heart Association. Circulation. 2015;132(22):2167-92.

6. Fouchier SW, Dallinga-Thie GM, Meijers JC, Zelcer N, Kastelein JJ, Defesche JC, Hovingh GK. Mutations in STAP1 are associated with autosomal dominant hypercholesterolemia. Circ Res. 2014;115(6):552-5.

7. Mikhailova S, Ivanoshchuk D, Timoshchenko O, Shakhtshneider E. Genes potentially associated with familial hypercholesterolemia. Biomolecules. 2019;9(12):807

8. Di Taranto MD, Giacobbe C, Fortunato G. Familial hypercholesterolemia: a complex genetic disease with variable phenotypes. Eur J Med Genet. 2020; 63(4):103831.

9. Ma J, Dempsey AA, Stamatiou D, Marshall KW, Liew CC. Identifying leukocyte gene expression patterns associated with plasma lipid levels in human subjects. Atherosclerosis. 2007:191(1):63-72.

10. Amor-Salamanca A, Castillo S, Gonzalez-Vioque E, Dominguez F, Quintana L, Lluis-Ganella C, Escudier JM, Ortega J, Lara-Pezzi E, Alonso-Pulpon L, et al. Genetically confirmed familial hypercholesterolemia in patients with acute coronary syndrome. J Am Coll Cardiol. 2017;70(14):1732-40

11. Cao YX, Wu NQ, Sun D, Liu HH, Jin JL, Li S, Guo YL, Zhu CG, Gao Y, Dong QT, et al. Application of expanded genetic analysis in the diagnosis of familial hypercholesterolemia in patients with very early-onset coronary artery disease. J Transl Med. 2018;16(1):345.

12. Braenne I, Kleinecke M, Reiz B, Graf E, Strom T, Wieland T, Fischer M, Kessler $T$, Hengstenberg C, Meitinger T, et al. Systematic analysis of variants related to familial hypercholesterolemia in families with premature myocardial infarction. Eur J Human Genet. 2016;24(2):191-7.

13. Danyel M, Ott CE, Grenkowitz T, Salewsky B, Hicks AA, Fuchsberger C, Steinhagen-Thiessen E, Bobbert T, Kassner U, Demuth I. Evaluation of the role of STAP1 in familial hypercholesterolemia. Sci Rep. 2019;9(1):1-8.

14. Sanchez-Hernandez RM, Tugores A, Novoa FJ, Brito-Casillas Y, ExpositoMontesdeoca AB, Garay P, Bea AM, Riano M, Pocovi M, Civeira F, et al. The island of gran Canaria: a genetic isolate for familial hypercholesterolemia. J Clin Lipidol. 2019;13(4):618-26.

15. Pirillo A, Garlaschelli K, Arca M, Averna M, Bertolini S, Calandra S, Tarugi P, Catapano AL, Group L. Spectrum of mutations in Italian patients with familial hypercholesterolemia: new results from the LIPIGEN study. Atheroscler Suppl. 2017;29:17-24.

16. lacocca MA, Wang J, Sarkar S, Dron JS, Lagace T, Mclntyre AD, Lau P, Robinson JF, Yang P, Knoll JH, et al. Whole-gene duplication of PCSK9 as a novel genetic mechanism for severe familial hypercholesterolemia. Can J Cardiol. 2018;34(10):1316-24

17. Lamiquiz-Moneo I, Restrepo-Cordoba MA, Mateo-Gallego R, Bea AM, Del Pino A-RM, Garcia-Pavia P, Cenarro A, Martin C, Civeira F, SanchezHernandez RM. Predicted pathogenic mutations in STAP1 are not associated with clinically defined familial hypercholesterolemia. Atherosclerosis. 2020;292:143-51.

18. Loaiza N, Hartgers ML, Reeskamp LF, Balder JW, Rimbert A, Bazioti V, Wolters JC, Winkelmeijer M, Jansen HPG, Dallinga-Thie GM, et al. Taking one step Back in familial hypercholesterolemia: STAP1 does not Alter plasma LDL (low-density lipoprotein) cholesterol in mice and humans. Arterioscler Thromb Vasc Biol. 2020;40(4):973-85.

19. White JK, Gerdin AK, Karp NA, Ryder E, Buljan M, Bussell JN, Salisbury J, Clare $S$, Ingham NJ, Podrini C, et al. Genome-wide generation and systematic phenotyping of knockout mice reveals new roles for many genes. Cell. 2013;154(2):452-64.

20. Bradley A, Anastassiadis K, Ayadi A, Battey JF, Bell C, Birling MC, Bottomley J, Brown SD, Burger A, Bult CJ, et al. The mammalian gene function resource: the international knockout mouse consortium. Mammalian Genome. 2012; 23(9-10):580-6.

21. Pettitt SJ, Liang Q, Rairdan XY, Moran JL, Prosser HM, Beier DR, Lloyd KC, Bradley A, Skarnes WC. Agouti C57BL/6N embryonic stem cells for mouse genetic resources. Nat Methods. 2009;6(7):493-5.

22. Ryder E, Gleeson D, Sethi D, Vyas S, Miklejewska E, Dalvi P, Habib B, Cook R, Hardy $M$, Jhaveri K, et al. Molecular characterization of mutant mouse strains generated from the EUCOMM/KOMP-CSD ES cell resource. Mammalian Genome. 2013:24(7-8):286-94.

23. Grippo RM, Tang Q, Zhang Q, Chadwick SR, Gao Y, Altherr EB, Sipe L, Purohit AM, Purohit NM, Sunkara MD, et al. Dopamine signaling in the Suprachiasmatic nucleus enables weight gain associated with hedonic feeding. Curr Biol. 2020;30(2):196-208.

24. Kuhel DG, Konaniah ES, Basford JE, McVey C, Goodin CT, Chatterjee TK, Weintraub NL, Hui DY. Apolipoprotein E2 accentuates postprandial inflammation and diet-induced obesity to promote hyperinsulinemia in mice. Diabetes. 2013:62(2):382-91.

25. Solca C, Tint GS, Patel SB. Dietary xenosterols lead to infertility and loss of abdominal adipose tissue in sterolin-deficient mice. J Lipid Res. 2013;54(2): 397-409.

26. Bjornstad P, Eckel RH. Pathogenesis of lipid disorders in insulin resistance: a brief review. Curr Diab Rep. 2018;18(12):127.

27. Chang Z, Huangfu C, Grainger AT, Zhang J, Guo Q, Shi W. Accelerated atherogenesis in completely ligated common carotid artery of apolipoprotein E-deficient mice. Oncotarget. 2017;8(66):110289-99.

28. Yang ZH, Gordon SM, Sviridov D, Wang S, Danner RL, Pryor M, Vaisman B, Shichijo Y, Doisaki N, Remaley AT. Dietary supplementation with long-chain monounsaturated fatty acid isomers decreases atherosclerosis and alters lipoprotein proteomes in LDLr(-/-) mice. Atherosclerosis. 2017;262:31-8.

29. Evans TD, Zhang X, Jeong SJ, He A, Song E, Bhattacharya S, Holloway KB, Lodhi IJ, Razani B. TFEB drives PGC-1alpha expression in adipocytes to protect against diet-induced metabolic dysfunction. Sci Signal. 2019;12(606): eaau2281. https://doi.org/10.1126/scisignal.aau2281.

30. Fritz BM, Munoz B, Yin F, Bauchle C, Atwood BK. A high-fat, high-sugar 'Western' diet alters dorsal striatal glutamate, opioid, and dopamine transmission in mice. Neuroscience. 2018;372:1-15.

31. Fouchier SW, Hutten BA, Defesche JC. Current novel-gene-finding strategy for autosomal-dominant hypercholesterolaemia needs refinement. J Med Genet. 2015:52(2):80-4

32. Taghizadeh E, Taheri F, Gheibi Hayat SM, Montecucco F, Carbone F, Rostami D, Montazeri A, Sahebkar A. The atherogenic role of immune cells in familia hypercholesterolemia. IUBMB Life. 2020;72(4):782-9.

\section{Publisher's Note}

Springer Nature remains neutral with regard to jurisdictional claims in published maps and institutional affiliations. 